\title{
Kinesin-1 captures RNA cargo in its adaptable coils
}

\author{
Jessica A. Cross, ${ }^{1,2}$ Derek. N. Woolfson, ${ }^{1,2,3}$ and Mark P. Dodding ${ }^{1}$ \\ ${ }^{1}$ School of Biochemistry, Faculty of Life Sciences, University of Bristol, Bristol BS8 1TD, United Kingdom; ${ }^{2}$ School of Chemistry, \\ Faculty of Life Sciences, University of Bristol, Bristol BS8 1TS, United Kingdom; ${ }^{3}$ Bristol BioDesign Institute, University of Bristol, \\ Bristol BS8 1TQ, United Kingdom
}

The prototypic and ubiquitous microtubule motor, kinesin-1, uses a variety of adaptor proteins to facilitate the selective transport of diverse cargo within the cell. These cargo adaptors bind to the motor complex through interactions with the kinesin light or heavy chains (KLCs or KHCs). In this issue of Genes \& Development, Dimitrova-Paternoga et al. (pp. 976-991) present the first structural characterization of a $\mathrm{KHC}$-cargo adaptor interface. They describe an antiparallel heterotrimeric coiled-coil complex between the carboxy tail of KHC and Tm1-I/C (aTm1), the atypical tropomyosin that is important for oskar mRNA transport in Drosophila oocytes. This interaction enhances direct binding between KHC and RNA. Their findings demonstrate the structural plasticity of the KHC tail as a platform for protein-protein interactions and reveal how a cargo adaptor protein can modify a motor-RNA interface to promote transport.

Kinesin-1 family microtubule motor proteins play a key role in intracellular transport in most cell types. They can engage diverse cargos including membrane-bound organelles (MBOs), proteins, and RNA, as well as many viruses and slide microtubules to control the organization of the microtubule network itself (Verhey and Hammond 2009; Lu and Gelfand 2017; Cross and Dodding 2019). To meet these complex functional requirements, kinesin-1 must specifically, and selectively, recognize cargos, and those interactions must be regulated. This is achieved in part through the binding of cargo adaptor proteins. These can be loosely defined as molecules that interact directly or indirectly with cargo and directly with a motor protein complex. As such, they define a key motor-cargo interface acting as bridges and regulatory hubs that control motor recruitment and activation. Recent structural studies have begun to show how these adaptors support selective MBO recognition via the KLCs /Cross and Dod-

[Keywords: kinesin; kinesin adaptor; kinesin-atypical tropomyosin complex; mRNA transport; oskar mRNA]

Corresponding author: mark.dodding@bristol.ac.uk

Article is online at http://www.genesdev.org/cgi/doi/10.1101/gad.348691. 121. Freely available online through the Genes \& Development Open Access option. ding 2019|, but much less is understood about how they enable recognition, recruitment, and transport of RNA.

Dimitrova-Paternoga et al. (2021) explore the role of the atypical tropomyosin, Tm1-I/C $(a \mathrm{Tm} 1)$, that is important for oskar mRNA localization to the posterior pole of the Drosophila oocyte. They solve X-ray crystal structures of an antiparallel homodimeric $a \mathrm{Tm} 1$ coiled coil in addition to a heterotrimeric complex consisting of two parallel KHCs and one antiparallel $a \mathrm{Tm} 1$ chain. The structure of the trimeric complex is validated by mutagenesis and biochemical and in vivo RNA transport assays. The KHC- $a \mathrm{Tm} 1$ complex is shown to bind RNA with higher affinity than KHC only, suggesting a new mechanism by which kinesin-cargo (RNA) transport can be modulated through stabilization by a KHC cargo adaptor. This is likely due to the positively charged binding surface formed in the trimeric KHC- $a \mathrm{Tm} 1$ complex and possible stabilization of an extended helical region of the KHC tail. To our knowledge, this is the first structural characterization of a direct cargo adaptor-KHC interface, giving important new insight into the mechanism of RNA recognition.

The region of KHC shown to bind $a \mathrm{Tm} 1$ immediately follows the sequences of KHC that are responsible for binding the KLCs (Fig. 1A,B; Diefenbach et al. 1998). This region also interacts with several other cargo adaptors and contains the ATP-independent microtubule binding site required for microtubule-microtubule sliding /Verhey and Hammond 2009; Lu and Gelfand 2017; Sanger et al. 2017). It will be important to explore whether and how the mode of binding $a \mathrm{Tm} 1$ extends to other adaptors and whether this influences motor-microtubule interactions. It is notable that RNA interaction with the KHC- $a \mathrm{Tm} 1$ complex is most likely enhanced due to presentation of an expanded positively charged binding surface; it seems possible that a related mechanism could also promote binding to the acidic tubulin C-terminal tails.

Previous studies have suggested that KLC is also important for Staufen/oskar localization (Lu et al. 2018) through interplay with another tetratricopeptide repeat protein, PAT1 (Loiseau et al. 2010). In addition to their role in

(C) 2021 Cross et al. This article, published in Genes \& Development, is available under a Creative Commons License (Attribution 4.0 International), as described at http://creativecommons.org/licenses/by/4.0/. 
A

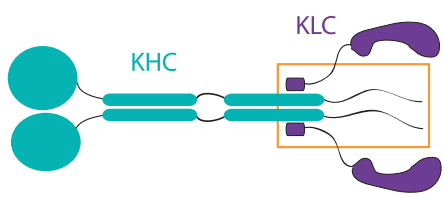

B
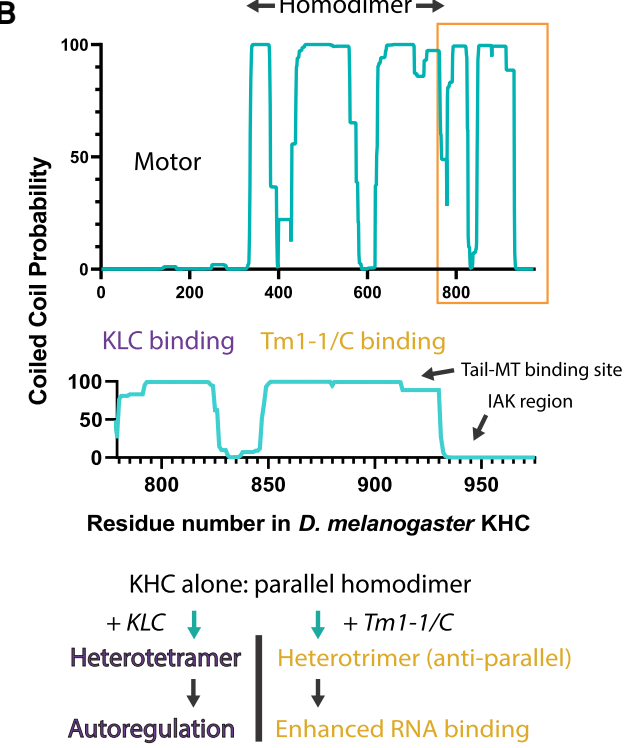

C

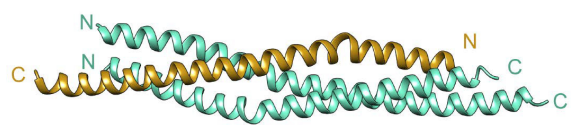

Figure 1. Structural and functional plasticity of the KHC coiled coils. (A) Schematic of the kinesin-1 heterotetramer. (Cyan) KHC, (purple) KLC. The orange boxed region highlights the KHC tail. (B, top) Marcoil (https://toolkit.tuebingen.mpg.de/tools/marcoil) coiled-coil prediction for $\mathrm{KHC}$; tail region is boxed orange. (Middle) Detailed coiled-coil prediction for the KHC tail that contains the binding sites for KLC and Tm1-I/C. (Bottom) Potential structural plasticity and associated function in the KHC coils. $(C)$ Crystal structure of the KHC-KHC-Tm1-I/C complex (PDB: 7BJS) from Dimitrova-Paternoga et al. (2021) (Cyan) KHCs, (brown) Tm1-I/C.

cargo recognition, KLCs are also important for mediating kinesin-1 autoinhibition, in a manner dependent on sequences containing their KHC binding heptad repeats (Verhey et al. 1998). Therefore, if some cargos are transported in a truly KLC-independent mechanism (i.e., KLC is not a component of the complex), this may suggest a secondary role for $\mathrm{KHC}$ tail-binding cargo adaptors such as $a \mathrm{Tm} 1$ in motor regulation, akin to that described for the KLCs. This seems plausible given that the $a \mathrm{Tm} 1$ binding site is sandwiched between the KLC binding site and the IAK region that interacts with the motor domains to mediate autoinhibition (Fig. 1B). However, a recent study in mammalian cells also mapping RNA/cargo adaptor binding determinants showed that SFPQ-RNA granules are transported by kinesin-1 tetramers comprised of KIF5A (a neuronal mammalian KHC paralog, also implicating the $\mathrm{KHC}$ tail in binding) and $\mathrm{KLC1}$, suggesting that the picture may be quite complex and could differ between RNA cargo and/or species (Fukuda et al. 2020).

Coiled coils most commonly form dimers, trimers, and tetramers in nature, with control over oligomeric state and orientation largely directed by patterns of isoleucine (Ile [I]), leucine (Leu [L]) in the core (heptad $a$ and $d$ positions), and salt bridges formed by adjacent residues ( $g$ and $e$ positions). As the investigators noted, the finding that the $a \mathrm{Tm} 1$ constructs crystallize as antiparallel coiled coils is in itself interesting; the received wisdom is that tropomyosins form exclusively parallel coiled-coil dimers (Hitchcock-DeGregori and Barua 2017). Therefore, for these constructs to behave differently from the norm is worth reflection. First, the $a$ and $d$ sites that define the hydrophobic part of the helix-helix interface are predominantly aliphatic hydrophobic with few obvious features that might discriminate between different coiled-coil structures. In addition, we note that the acidic and basic side chains at the $g$ and $e$ positions (Fig. 4A in Dimitrova-Paternoga et al. 2021) could possibly be better accommodated in a parallel arrangement. Thus, it is possible that these regions of the $a \mathrm{Tm} 1$ sequence are somewhat promiscuous or agnostic with regard to coiled-coil partner selection and orientation. In turn, this could contribute to its adaptability as the investigators elegantly and persuasively show. In contrast to $a \mathrm{Tm} 1$, the two KHCs retain a parallel interaction in the complex (Fig. 1C). Nonetheless, to form a trimeric hydrophobic core with the additional $a \mathrm{Tm} 1$ helix, the KHC coils must also show conformational flexibility to open up the interface. This must also be true of the adjacent KLC binding site, where the KHCs presumably undergo a transition from a homodimeric to heterotetrameric coiled-coil assembly to accommodate the KLC heptad repeats (Fig. 1B). It is not clear whether this interaction is parallel or antiparallel. It will be interesting to discover whether KLC heterotetramer and cargo adaptor heterotrimer states can occur simultaneously.

Together, these data form a picture of the KHC stalk as a dynamic and flexible platform for protein-protein interactions. This is in contrast to the rigid spacer-like properties with functions limited to oligomerization sometimes associated with coiled-coil domains. This could play an important role in the larger conformational changes associated with transition from a compact autoinhibited state to an extended active form of the motor, capable of motility on the cytoskeleton. DimitrovaPaternoga et al. (2021) provide important molecular insight into how RNA cargos are recognized by this crucial molecular machine. Their findings suggest that it is now time for the often-neglected kinesin-1 coiled coils to move to the fore as we expand our understanding of regulation and its coupled, remarkably versatile, cargo selection mechanisms.

\section{Acknowledgments}

M.P.D. is a Lister Institute of Preventative Medicine Fellow, and work in his laboratory is supported by the Biotechnology and 
Biosciences Research Council (BB/S000917/1). J.A.C. is supported by the Engineering and Physical Sciences Research Council Bristol Centre for Doctoral Training in Chemical Synthesis.

\section{References}

Cross JA, Dodding MP. 2019. Motor-cargo adaptors at the organelle-cytoskeleton interface. Curr Opin Cell Biol 59: 16-23. doi:10.1016/j.ceb.2019.02.010

Diefenbach RJ, Mackay JP, Armati PJ, Cunningham AL. 1998. The C-terminal region of the stalk domain of ubiquitous human kinesin heavy chain contains the binding site for kinesin light chain. Biochemistry 37: 16663-16670. doi:10.1021/ bi981163r

Dimitrova-Paternoga L, Jagtap PKA, Cyrklaff A, Vaishali, Lapouge K, Sehr P, Perez K, Heber S, Löw C, Hennig J, et al. 2021. Molecular basis of mRNA transport by a kinesin-1atypical tropomyosin complex. Genes Dev (this issue). doi:10.1101/gad.348443.121

Fukuda Y, Pazyra-Murphy MF, Silagi ES, Tasdemir-Yilmaz OE, Li Y, Rose L, Yeoh ZC, Vangos NE, Geffken EA, Seo H-S, et al. 2020. Binding and transport of SFPQ-RNA granules by KIF5A/KLC1 motors promotes axon survival. I Cell Biol 220: e202005051. doi:10.1083/jcb.202005051

Hitchcock-DeGregori SE, Barua B. 2017. Tropomyosin structure, function, and interactions: a dynamic regulator. In Fibrous proteins: structures and mechanisms (ed. Parry DAD, Squire JM), pp. 253-284. Springer International Publishing, Cham.

Loiseau P, Davies T, Williams LS, Mishima M, Palacios IM. 2010. Drosophila PAT1 is required for kinesin- 1 to transport cargo and to maximize its motility. Development 137: 2763-2772. doi:10.1242/dev.048108

Lu W, Gelfand VI. 2017. Moonlighting motors: kinesin, dynein, and cell polarity. Trends Cell Biol 27: 505-514. doi:10.1016/j .tcb.2017.02.005

Lu W, Lakonishok M, Serpinskaya AS, Kirchenbüechler D, Ling S-C, Gelfand VI. 2018. Ooplasmic flow cooperates with transport and anchorage in Drosophila oocyte posterior determination. I Cell Biol 217: 3497-3511. doi:10.1083/jcb .201709174

Sanger A, Yip YY, Randall TS, Pernigo S, Steiner RA, Dodding MP. 2017. SKIP controls lysosome positioning using a composite kinesin-1 heavy and light chain-binding domain. I Cell Sci 130: 1637-1651.

Verhey KJ, Hammond JW. 2009. Traffic control: regulation of kinesin motors. Nat Rev Mol Cell Biol 10: 765-777. doi:10 $.1038 / \mathrm{nrm} 2782$

Verhey KJ, Lizotte DL, Abramson T, Barenboim L, Schnapp BJ, Rapoport TA. 1998. Light chain-dependent regulation of kinesin's interaction with microtubules. J Cell Biol 143: 10531066. doi: $10.1083 /$ jcb.143.4.1053 


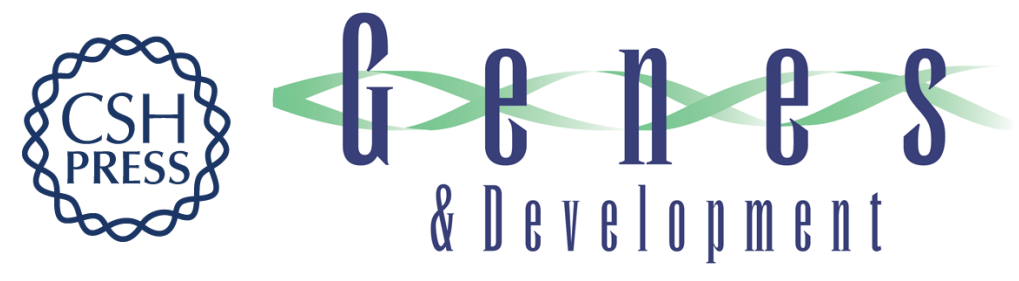

\section{Kinesin-1 captures RNA cargo in its adaptable coils}

Jessica A. Cross, Derek. N. Woolfson and Mark P. Dodding

Genes Dev. 2021, 35:

Access the most recent version at doi:10.1101/gad.348691.121

Related Content Molecular basis of mRNA transport by a kinesin-1atypical tropomyosin complex Lyudmila Dimitrova-Paternoga, Pravin Kumar Ankush Jagtap, Anna Cyrklaff, et al. Genes Dev. July , 2021 35: 976-991

References This article cites 10 articles, 5 of which can be accessed free at: http://genesdev.cshlp.org/content/35/13-14/937.full.html\#ref-list-1

Articles cited in: http://genesdev.cshlp.org/content/35/13-14/937.full.htmI\#related-urls

Creative This article, published in Genes \& Development, is available under a Creative Commons Commons License License (Attribution 4.0 International), as described at http://creativecommons.org/licenses/by/4.0/.

Email Alerting Receive free email alerts when new articles cite this article - sign up in the box at the top Service right corner of the article or click here.

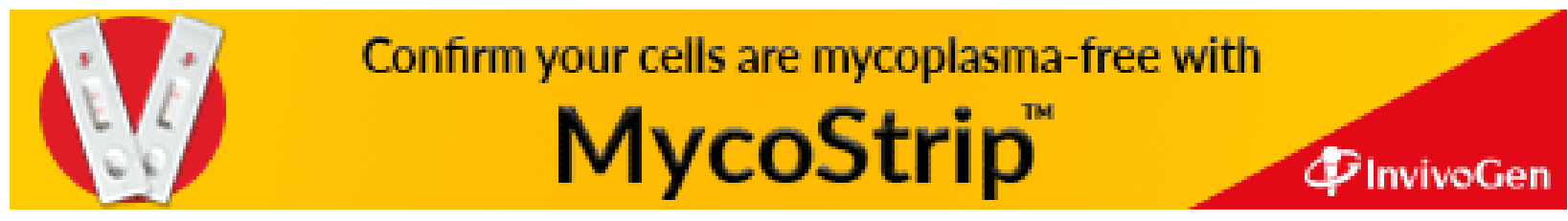

